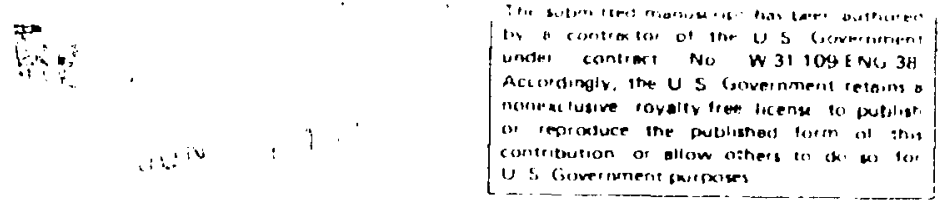

PHYSICS IN THE GeV REgION WITH POLARIZED TARGETS IN

\title{
ELECTRON STORAGE RINGS
}

\author{
Roy J. Holt \\ Physics Division \\ Argonne National Laboratory \\ Argonne, IL 60439-4843
}

CONF-880386--7

DE88 011966

\section{INTRODUCTION}

During the past five years interest in the internal target method for electron storage rings has intensified. At present there is an active internal target program at the VEPP-3 ring in Novosibirsk and a second ring (NEP) dedicated for nuclear physics is under construction there. An internal Ar jet target is in use at the ADONE ring in Frascuti in order to produce tagged photons. In addition, plans are being made for an internal target program at the $300-\mathrm{MeV}$ electron ring at Saskatoon. Construction of electron rings at MIT-Bates and NIKHEF is beginning and both laboratories plan internal target facilities. Finally, there are discussions with regard to the PF.P and HERA rings for internal target experiments in the Multi-GeV region.

Perhaps the least discussed advantage of the internal target method is the enormous dynamic range available in electron energy. The energy range for experiments varies from $0.22 \mathrm{GeV}$ at the NEP ring to $30 \mathrm{GeV}$ at HERA. Thus, the internal target method is beneficial to a full program of nuclear physics research. For example, a program to study collective modes in the nuclens is planned for the NEP ring, while physics discussions in the GeV region are centered around the emerging fields of nuclear chromocynamics and the effects of color transparency. Since Argonne has embarked upon a joint venture with the licyssibirsk group to measure the tensor analyzing power $\mathrm{T}_{20}$ in electron-deuteron elastic scattering at the $2-\mathrm{GeV}$ VEPP-3 ring, I shall draw upon examples from this energy region for the discussion today. Finally, there is interest in the study of the structure of the nucleon at the HERA ring in which it is envisaged that polarized electrons and targets will be used to measure the spin structure functions of the proton and neutron.

\section{DEUTERON PHOTODISINTEGRATION IN THE GeV REGION}

It is particularly interesting to consider the two-body breakup of the deuteron in the $\mathrm{GeV}$ region for three reasons: (i) the electromagnetic interaction with the simplest nucleus, the deuteron, is especially amenable to theoretical interpretation, (ii) at high energy one is probing the short-range part of the deuteron wave function, and (iii) in this energy region there are considerable differences between the predictions of meson-exchange theory 1 and nuclear chromodynamics.

Two hypotheses are inherent within the framework of nuclear chromodynamics: the overall energy dependence of the cross section is known from arguments of dimensional scaling and $Q C D$; and the form factors of the 
two nucieors are factorizable. Thus, the expected energy dependence of the' differential cross section has the form

$$
\frac{d \sigma}{d n} \propto \frac{1}{\left[s\left(s-m_{d}^{2}\right)\right] 1 / 2} F_{p}^{2}\left(t_{p}\right) F_{n}^{2}\left(t_{n}\right) \frac{f^{2}\left(\theta_{c m}\right)}{p_{T}^{2}}
$$

where $s$ is the square of the total energy in the center-of-mass frame, $\mathrm{m}_{d}$ is the deuteron mass, $p_{\eta}$ is the transverse momentum and $F(p)$ are the proton (or neutron) electromagnetic form factors. Here, $f\left(\theta_{\mathrm{cm}}\right)$ is the reduced amplitude which is expected to have no energy dependence where the nuclear chromodynamic description is valid. This reduced amplitude has been deduced for existing data at $\theta_{\mathrm{cm}}=90^{\circ}$ and is illustrated in Fig. 1 . Also shown in Fig. 1 is a recent meson-exchange calculation of T.-S. H. Lee. New and preliminary data, illustrated as the solid circles in the figure, are from experiment ${ }^{3,4}$ NE8 at SLAC. Clearly, the energy dependence of new data are better described from the simple arguments of nuclear chromodynamics than by the meson-exchange theory above $1 \mathrm{GeV}$. The analysis of these data is preliminary in that the absolute normalization was taken from an on-line analysis of electron-proton elastic scattering. However, the energy dependence of the results is not expected to change significantly in the final analysis. Polarization experiments should have an important role in the GeV region as a further test of this finding.

\section{ELECTRON-DEUTERON ELASTIC SCATTERING}

M. Garcon in the previous talk ${ }^{5}$ gave a thorough introduction to the motivation for a measurement of $\mathrm{T}_{20}$ in electron-deuteron elastic scattering, and I shall not repeat it here. Briefly, the major issues are: (i) the first isolation of the charge and quadrupole form factors $f \circ$ a the deuteron, (ii) investigation of $\mathrm{QCD}$ effects, and (iii) information on the little understood isoscalar meson-exchange current. In fact, it is expected that a measurement

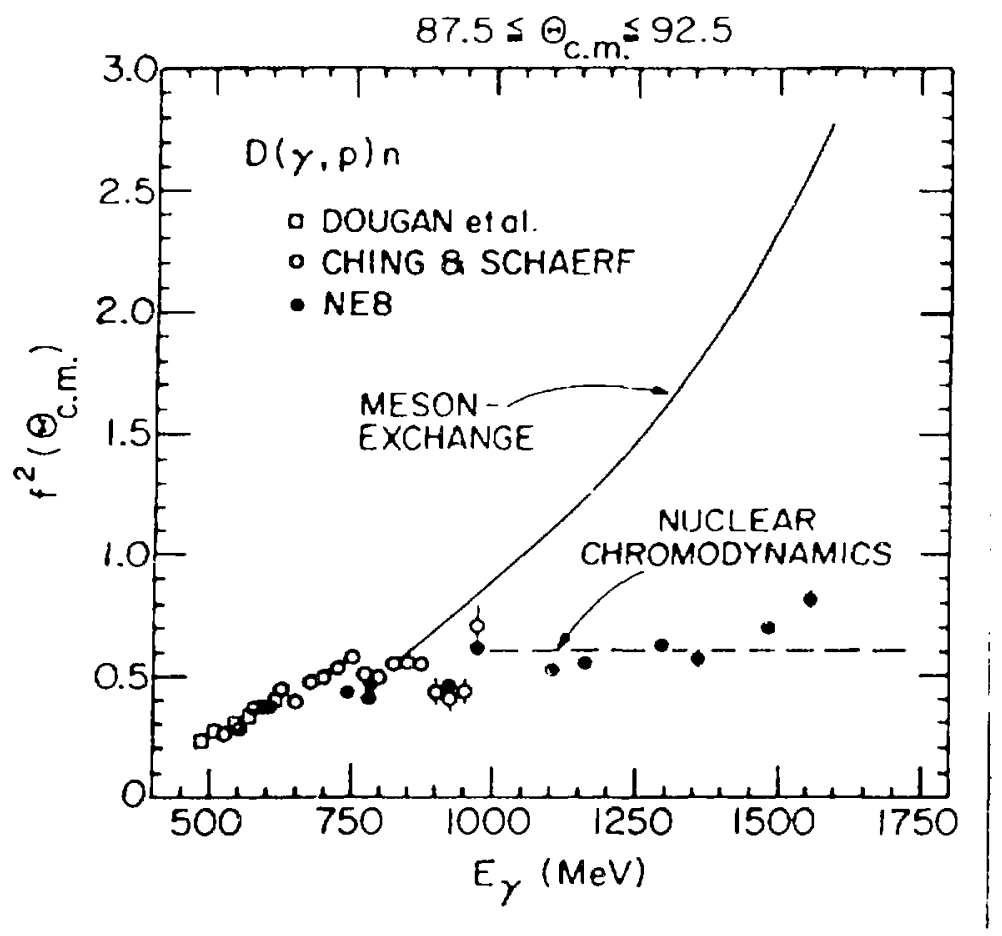

Fig. 1 The solid curve indicates the prediction of the meson-exchange model while the dashed curve is that of the nuclear chromodynamics model. The new data from experiment NE8 at SLAC are given by the solid circles. Note that these data are based upon a preliminary analysis, as described in the text. 


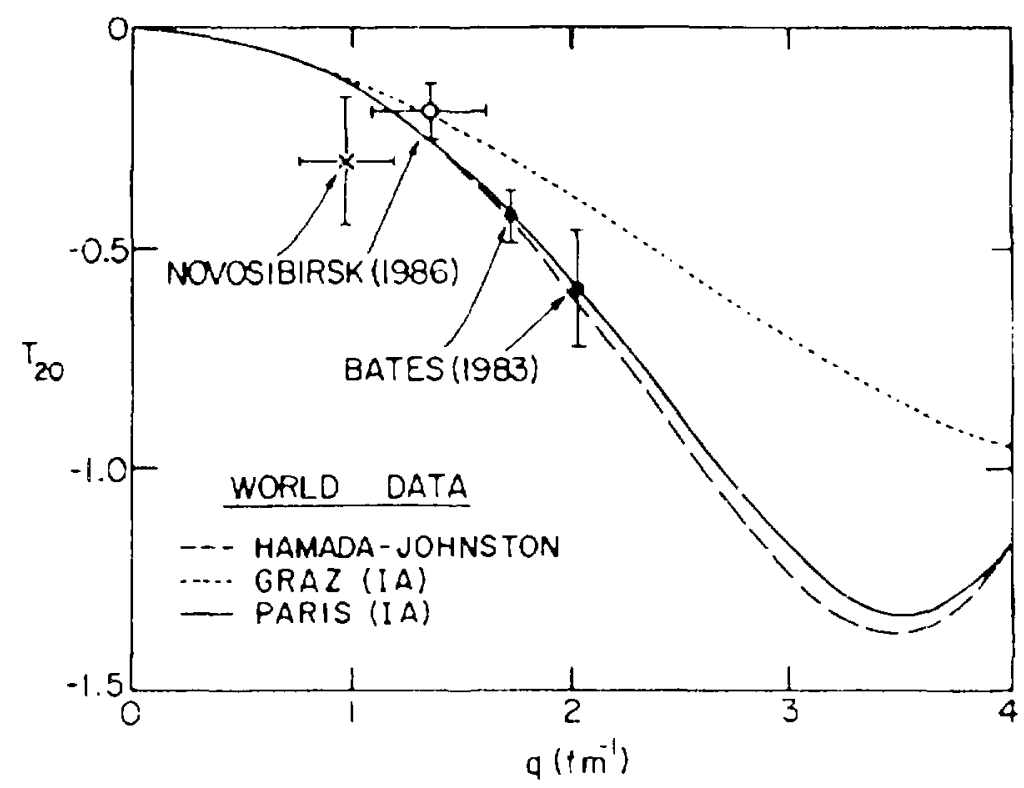

Fig. 2 Summary for all polarization data in electron-deuteron elastic scattering. The two data points at low values of $\mathrm{q}$ were determined with an internal polarized target method while the two higher $q$ points were measured with a deuteron polarimeter.

of $\mathrm{T}_{20}$ at high momentum transfer would be the most sensitive test of $\mathrm{QCD}$ effects in nuclei, since $T_{20}$ is expected to approach a value of $-\sqrt{2}$ in the QCD limit. All existing data for polarization in electron-deuteron scattering are illustrated in Fig. 2. Note that this graph represents all existing data for polarization in electron scattering from nuclei. The two data points at high momentum transfer were measured with a deuteron tensor polarimeter at the MIT-Bates Laboratory, while the lower momentum transfer data ${ }^{7}$ were determined with an internal polarized target at Novosibirsk. Clearly, these measuxements must be extended to high momentum transfer and efforts are underway both at MIT and Novosibirsk.

A comparison of the relative scales of these two experiments is indicated schematically in Fig. 3. The experiment at MIT required the use of two large magnetic spectrometers and tra attendant shielding to detect the scattered electrons and deuterons; whereas, the internal target experiment (shown approximately on the same scale in the inset figure) was performed with detector arrays. The Novosibirsk apparatus also is shown with a magnified scale so that the individual components can be seen. The detector hardware is simplified in the internal target geometry because of the purity of the polarized target, the high duty factor of the ring and the low background from the thin internal target.

Of course, the primary limitation of the injernal target geometry is the relativiely small target thickness, $10^{11}$ nuclei $/ \mathrm{cm}^{2}$. Efforts are underway ${ }^{8}$ at Argonne to produce a high flux, laser-driven polarized $H$ or $D$ source and to develop a storage cell for the polarized atoms in order to increase the thickness of the polarized target.

\section{ARGONNE-NOVOSIBIRSK COLLABORATION}

The news today is that Argonne and Novosibirsk have embarked upon a collaborative effort in order to measure the tensor analyzing power $T_{20}$ in electron-deuteron scattering at the highest possible momentum transfer. This collaboration was initiated last month when $L$. Young and I brought to Novosibirsk a secially fabricated storgge cell for polarized atoms in the VEPP-3 storage ring. This collaboration is based upon the experience of the 


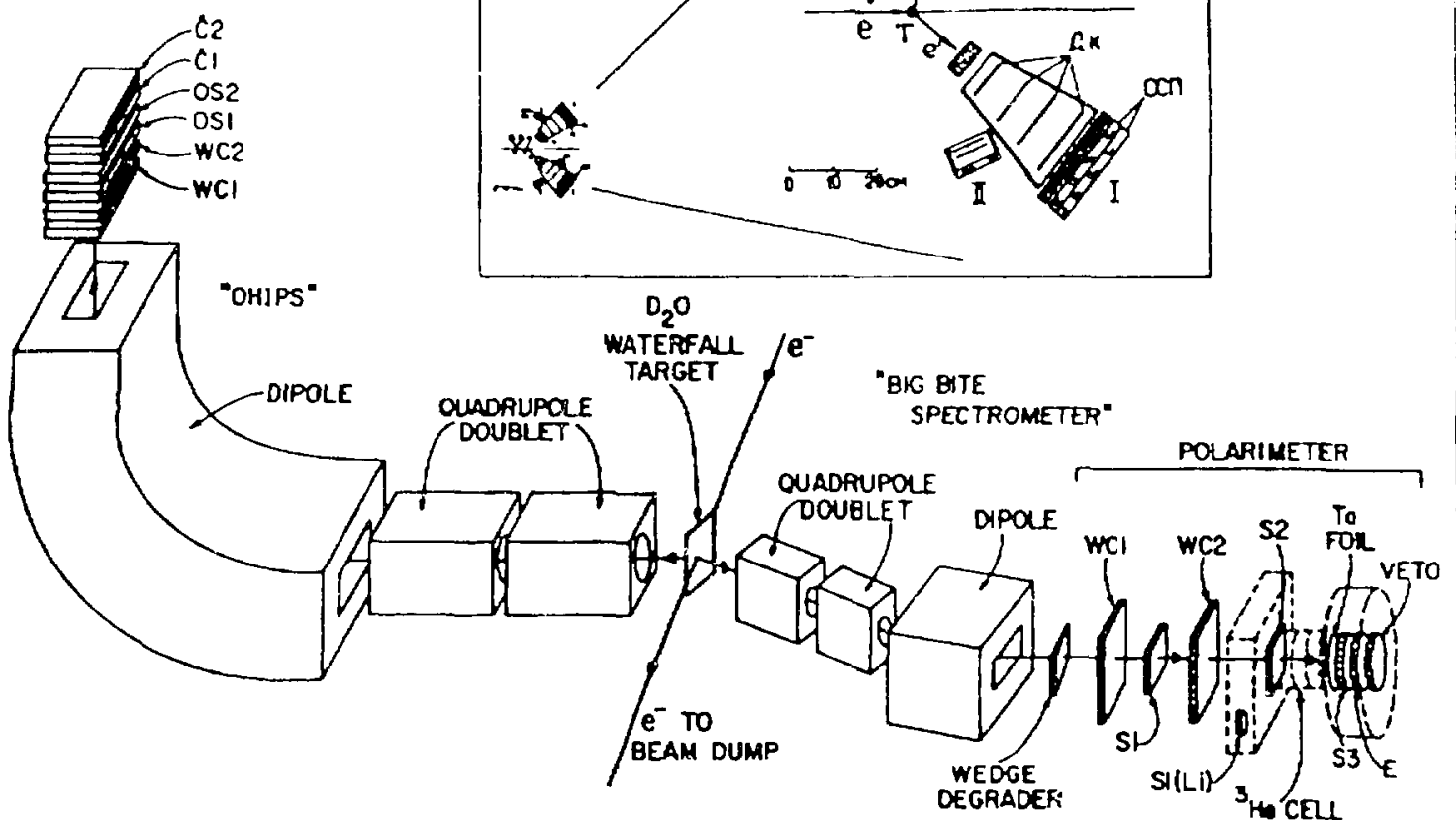

Fig. 3 Schematic diagram of the apparatus required to measure $t_{20}$ in $e-d$ elastic scattering in the external target geometry. Inset figure on left illustrates the apparatus on approximately the same scale for the internal target experiment at Novosibirsk. The figure is also shown with an expanded scale for ease of identification of the components.

Novosibirsk group in the technology of ultra-thin internal targets in electron storage rings and the experience of Argonne in the development of storage cells for polarized atoms and laser-driven polarized atomic beam sources. Presently, it is perceived that the collaborative effort will proceed in three phases in order to achieve the necessary target thickness and luminosity: (i) use of the Novosibirsk polarized source and a simple ANL storage cell ( $n=$ $\left.1 \times 10^{12} \mathrm{~cm}^{-2}\right)$, (ii) use of the Novogibirsk source and a more sophisticated clam shell storage cell $\left(\mathrm{n}=1 \times 10^{15} \mathrm{~cm}^{-2}\right)$, (iij) replace the Novosibirsk source with the ANL laser-driven source $\left(n=1 \times 10^{\left.1 / \mathrm{cm}^{-2}\right)}\right.$.

With regard to storage cells for polarized atoms, the central issue is the surface coating of the cell. A drifilm coating was used for the initial simple ANL storage fell which presently resides in the VEPP-3 ring. Drifilm, the most studied 8 surface coating, has the properties that it chemically bonds to the surface and thereby is bakeable at relatively high temperatures. $A$ teflon surface is also expected ${ }^{11}$ to inhibit depolarization of $H$ and $D$ atoms, but it is not alkali resistant and cannot be used with the laser-driven source.

\section{INTERNAL TARGET DEVELOPMENT}

Now I would like to focus on some recent developments in laser-driven polarized $H, D$ and ${ }^{3}$ He targets. A high-flux polarized source of $H$ and $D$ atoms based upor an optical-pumping spin-exchange technique is being developed at Argonne. The method involves a two-step process in which an ensemble of alkali atoms, $K$ in the present case, are polarized by optical pumping and the polarization is transferred to $H$ or $D$ atoms via spinexchange scattering. This method is attractive since the flux of $H$ or $D$ 

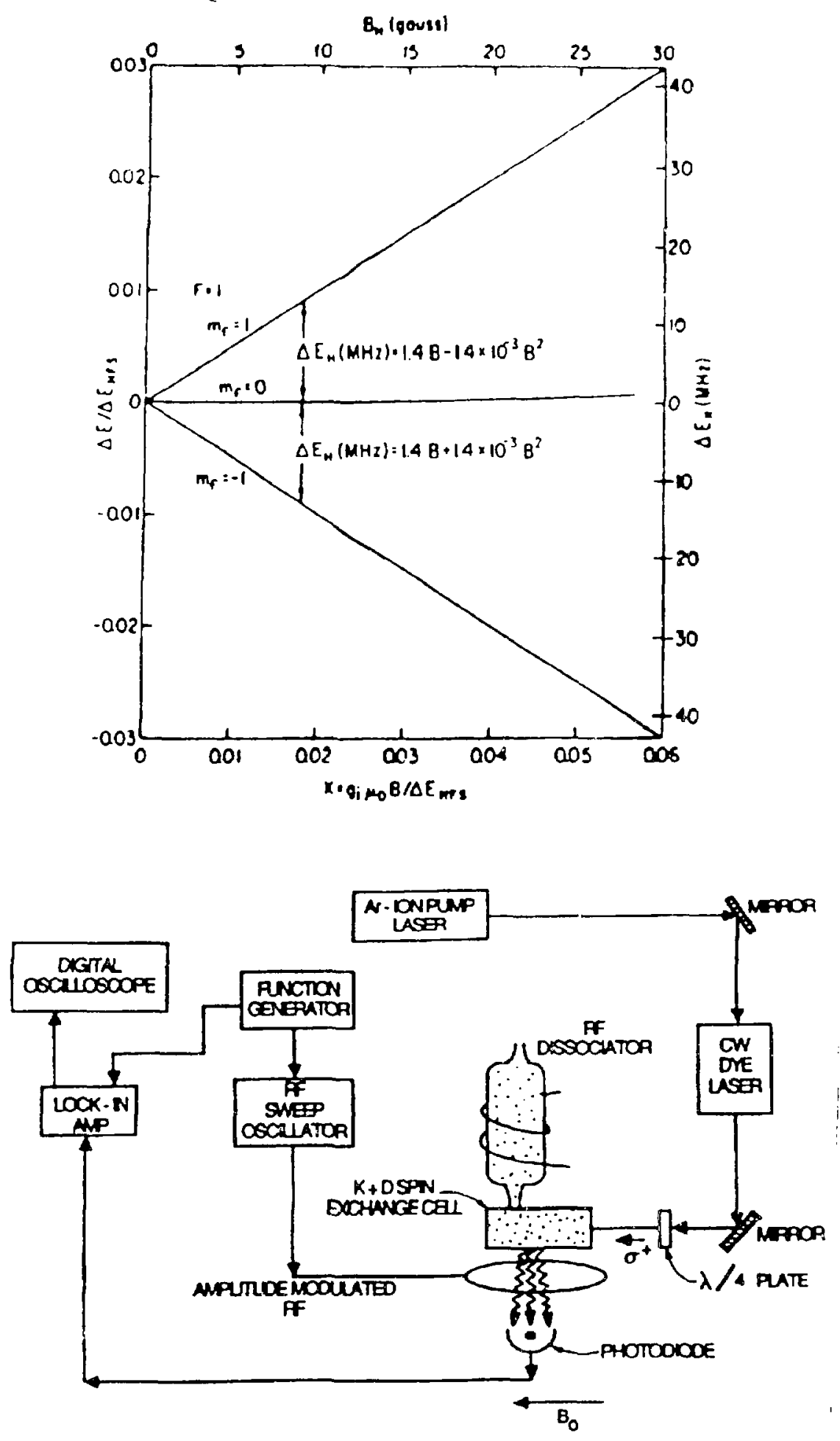

Fig. 4 Breit-Rabi diagram for the $H$ atoms indicating the two available Zeeman transitions. The apparatus to induce and detect these Zeeman transitions is illustrated schernatically. 


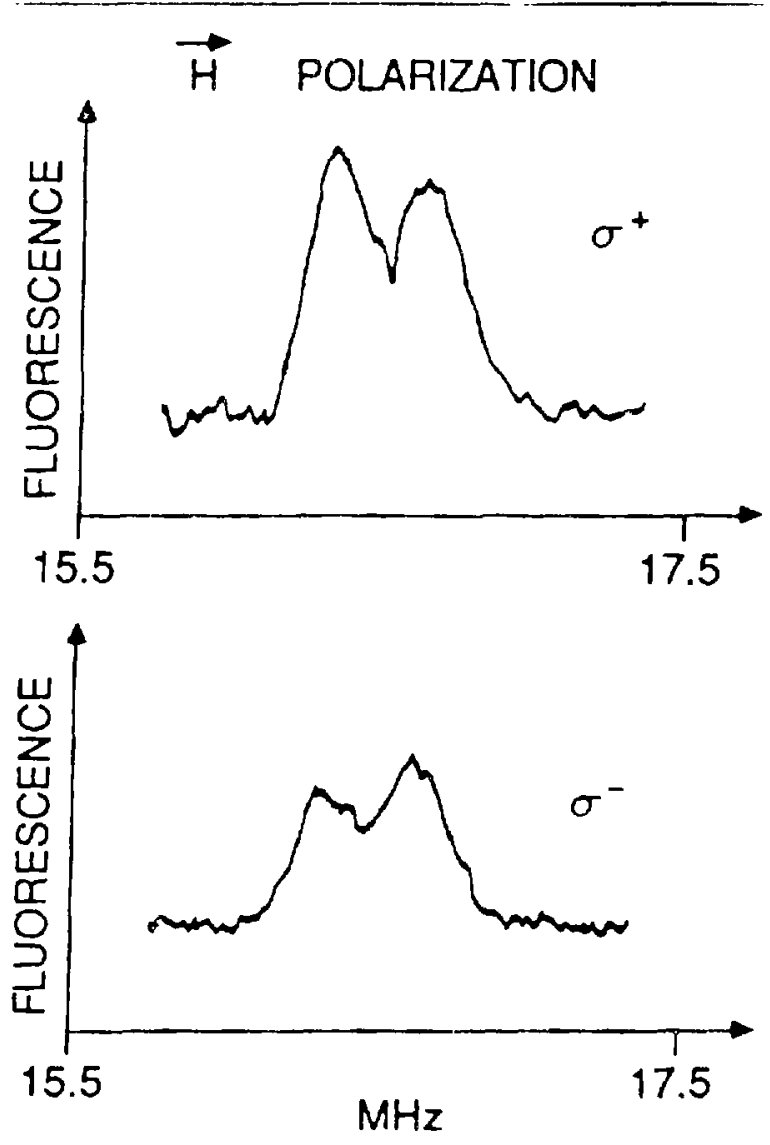

Fig. 5 A comparison of Zeeman transitions for $H$ atoms for $\sigma^{+}$and $\sigma^{-}$laser light incident on the spin-exchange cell. In this case the $H$ polarization is $10 \%$ and the flux is $6 \times 10^{16} \mathrm{~s}^{-1}$. These results represent the first observation of polarized $H$ from a spin-exchange optically-pumped source.

atoms is limited, in principle, by available laser power and since the gas load to a storage ring would be minimal.

The primary technical challenge is effective optical-pumpirg of the $K$ atoms without a buffer gas. This means that the spectrum of the laser light must be carefully matched to the Doppler-broadened line width of the $K$ atoms and that the surface in the spin-exchange ce!l must inhibit spin. relaxation of the $K$ and $H$ atoms. The polarization was determined by observing fluorescence from $K$ atoms in the spin-exchange cell when the Zeeman transition frequency for the $H$ atoms was swept wiin an RF coil. A Breit-Rabi diagram for the $H$ atom is shown in the upper panel of Fig. 4, while the apparatus is shown schematically in the lower half of the figure. If the $H$ atoms are partially polarized we expect to observe two Zeeman transitions in the intermediate magnetic fieid region. These transitions are observed and shown in Fig. 5. Clearly, the relative intensity of the two transitions reverses as expected between $\sigma^{+}$and $\sigma^{-}$light. Here we see the first evidence for $H$ from an optically-pumped, spin-exfhange source! Thus far, a vector polarization of $20 \%$ with a flux of $1.3 \times 10^{17 \mathrm{~s}^{-1}}$ for the $\mathrm{H}$ atoms has been achieved. With the assumption that the $H$ atoms are in equilibrium with the $K$ atoms, and consequently, that the populations $N\left(p_{m}^{p}\right)$ of the atoms in $H$ follow a spin-temperature distribution, i.e. $N\left(m_{F}\right)$ a where $\rho$ is the spin temperature and $m_{F}$ is the total magnetjc quantum number, the polarization of the protons in the spin-exchange cell is found to be $10 \%$ from these data. Further improvement in the polarization is expected as we gain more experience with the source. 

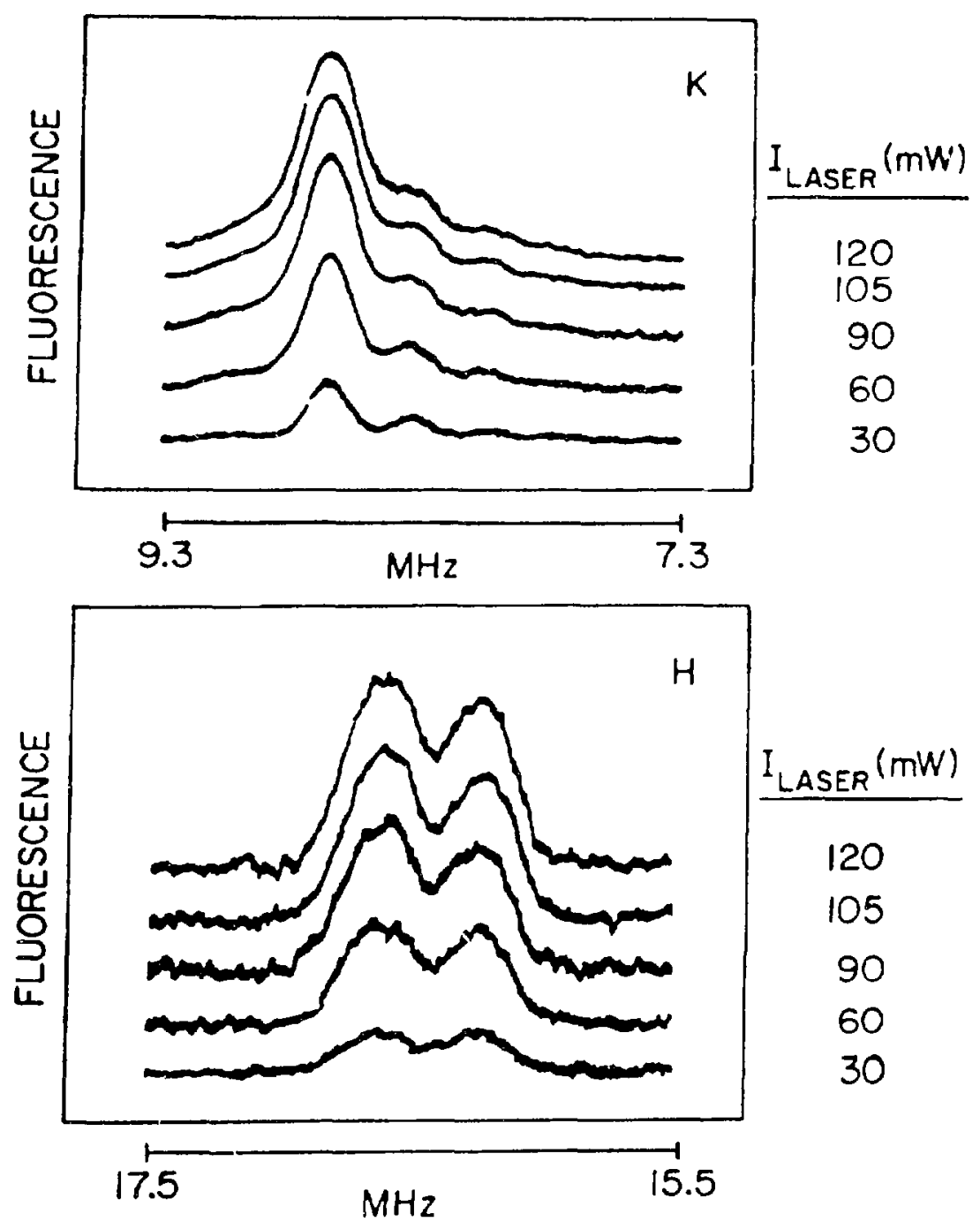

Fig. 6 Zeeman transition signals for $K$ (upper panel) and $H$ (lower panel) ds a function of incident laser power.

Since these first Zeeman signals were observed the laser light was more carefully matched to the Doppler spectrum of the $K$ atoms. The typical Zeeman signals now observed are shown in Fig. 6 for $K \uparrow$ in the upper panel and $\mathrm{H} \uparrow$ in the lower panel. Clearly, the signals improve as the laser power is increased. In fact, the next step based on these studies is to increase the laser power by adding a second $\mathrm{CW}$ dye laser.

The goal is to provide a target of $D$ atoms with a thickness of $10^{14}$ $\mathrm{cm}^{-2}$ and a tensor polarization of $t_{20} \geqslant 0.3$ which could be used in an electron-deuteron scattering experiment. Again, in order to achieve this thickness it is essential to store the atoms in a windowless cell and permit the atoms to leak away before the spins relax. In order to achieve this goal the high flux and polarization expected from the laser-driven source is essential.

A novel internal polarized ${ }^{3} \mathrm{He}$ target is being developed ${ }^{12}$ at Caltech. The ${ }^{3 e}$ nuclei are polarized by the well-known method of optically-pumping metastable ${ }^{3} \mathrm{He}$ atoms. This method has benefitted recently figm the development of lasers which can operate at $1.083 \mu \mathrm{m}$ so that $\delta 3 \times 10^{18} \mathrm{H} \mathrm{He} / \mathrm{s}$ are produced. It is expected that an internal target of $\mathrm{fe}$ with a thickness of $>10^{15} \mathrm{~cm}^{-2}$ is feasible. 
In summary, it appears that we have evidence from the $D(\gamma, p) n$ reaction that the meson-exchange model is failing in the $\mathrm{GeV}$ region. Surprisingly, it appears that the new $D(\gamma, p) n$ data favor the energy dependence of the nuclear chromodynamics model rather that that of the meson-exchange model.

Application of the polarization method to electron scattering studies is in its infancy, and it is potentially a very powerful technique. The internal target method coupled with laser-driven polarized targets should represent an important tool for nuclear physics.

\section{ACKNOWLEDGEMENTS}

I wish to thank those who have contributed most significantly to the polarized target development: L. Young, M. Green, R. Kowalczyk and J. Gregar.

This work supported by the U. S. Department of Energy, Nuclear Physics Division, under contract W-31-109-ENG-38.

\section{REFERENCES}

1. T.-S. H. Lee, to be published.

2. S. Brodsky and J. Hiller, Phys. Rev. C 28, 475 (1983).

3. J. Napolitano, et al., to be published.

4. The NE8 collaboration is: S. J. Freedman, D. F. Geesaman, R. Gilman, M. C. Green, R. J. Holt, H. E. Jackson, E. Kinney, C. Marchand, J. Napolitano, B. Zeidman, R. E. Segel, T.-Y. Tung, P. Bosted, D. Beck, G. Boyd, D. Collins, B. Filippone, J. Jourdan, R. D. McKeown, R. Milner, D. Potterveld, R. Walker, C. Woodward, Z.-E. Meziani, and R. Minehart.

5. M. Garcon, Proceedings of this Conference.

6. M. E. Schulze, et al., Phys. Rev. Lett. 52, 597 (1984).

7. B. B. Woitsekhowski, et al., JETF Lett. 43, 567 (1986).

8. L. Young, et al., Nucl. Instrum. and Meth. B24/25, 963 (1987).

9. The collaborators at Novosibirsk are presently: S. G. Popov, B. B. Woitsekhowski, D. M. Nikolenko, D. K. Torpokov, E. P. Tsentalovich, I. R. Rachek, V. F. Dmitriev, V. G. Zelevinsky, and M. Mostovoy; and at ANL: R. J. Holt, R. Gilman, L. Young, and R. Kowalczyk.

10. D. R. Swenson and L. W. Anderson, Nucl. Instrum. and Meth. B29, 627 (1988); J. S. Camparo, J. Chem. Phys. 86, 533 (1987); H. M.' Goldenberg, et al., Phys. Rev. Lett. 5, 361 (1960).

11. W. Haeberli, Bull. Am. Phys. Soc. 31, 1195 (1986); T. Wise, private communication.

12. R. G. Milner, et al., Nucl. Instrum. and Meth. A257, 286 (1987); C. L. Bohler, et al., to be published.

\section{DISCLAIMER}

\footnotetext{
This report was prepared as an account of work sponsored by an agency of the linited States Government. Neither the United States Government nor any agency thereof, nor any of their employees, makes any warranty, express or implied, or assumes any legal liability or responsibility for the accuracy, completeness, or usefulness of any information, apparatus, product, or process disclosed, or represents that its use would not infringe privately uwned rights. Reference herein to any specific commercial product, process, or service by trade name. tradcmark. manufacturer, or otherwise does not neccssarily constitute or imply its endorsement, recommendation, or favoring by the United States Government or any agency thereof. The vews and opinions of authors expressed herein do not necessarily state or reflect those of the United States Government or any agency thereof.
} 